\title{
Traumatic recurrent distal radioulnar joint dislocation: a case report
}

\author{
Sander Wassink • Lukas A. Lisowski • \\ Bernard G. Schutte
}

Received: 6 July 2009/Accepted: 11 November 2009/Published online: 24 November 2009

(C) Springer-Verlag 2009

\begin{abstract}
Isolated acute distal radioulnar joint (DRUJ) dislocation is a rare injury (Garrigues and Aldridge III in $\mathbf{J}$ Bone Joint Surg Am 89:1594-1597, 2007]. Reports of isolated DRUJ luxations, volair or dorsal, are often case reports and rarely a series of cases [Dameron Jr in Clin Orthop Relat Res 83:55-63, 1972]. We present a case of an acute traumatic dorsal DRUJ dislocation treated with cast immobilization with recurrence of the dislocation after a new trauma some months later. At follow-up, 17 months after the first dislocation and 9 months after the second, he experienced no pain and had no restrictions in work or sports-related activities.
\end{abstract}

Keywords Distal radioulnar joint dislocation .

Recurrent DRUJ luxation - Conservative treatment

\section{Introduction}

Isolated acute distal radioulnar joint (DRUJ) dislocation is a rare injury [1]. It is usually associated with fractures of either the radius or ulna. Sometimes it is part of a more complex injury like the Essex-Lopresti injury in which a

S. Wassink - B. G. Schutte

Spaarne Hospital, Spaarnepoort 1, 2134 TM Hoofddorp,

The Netherlands

L. A. Lisowski

Academic Medical Center, Meibergdreef 9,

1105 AZ Amsterdam, The Netherlands

S. Wassink $(\bowtie)$

Bloemhofstraat 9 zwart, 2012 ZV Haarlem,

The Netherlands

e-mail: wassinksander@hotmail.com fracture of the proximal radius and rupture of the interosseus membrane occurs. Reports of isolated DRUJ luxations, palmar or dorsal, are often case reports and rarely a series of cases [2]. We present a case of an acute traumatic dorsal DRUJ dislocation treated with cast immobilization with recurrence of the dislocation after a new trauma some months later.

\section{Case report}

A 20-year-old man presented himself to our emergency department 4 days after a fall in the bathtub. The exact mechanism of the injury was not clear. He reported persistent pain in the right wrist and the inability to rotate his hand with the palmar side up.

At physical examination, the wrist was swollen dorsally with a small hematoma located at the distal ulna. Pronation was not restricted, whereas supination was impossible beyond the neutral position. The X-ray revealed a dorsally located ulna in correlation to the radius. X-rays from the unaffected side were taken for comparison (Fig. 1).

Reposition was attempted under conscious sedation by pressure on the ulna and a gently forced supination, but was not successful. Under general anesthesia, the dislocation could be reduced, and an above elbow cast in full supination was applied. Postoperative X-rays showed a normal relation between the ulna and radius. After 4 weeks, the cast was replaced by a fore-arm cast for two more weeks. Six weeks after dislocation, the patient experienced no pain but still had a limited supination of $50^{\circ}$ (Fig. 2).

Eight months after the first trauma, he presented himself to our emergency department with pain in the right 

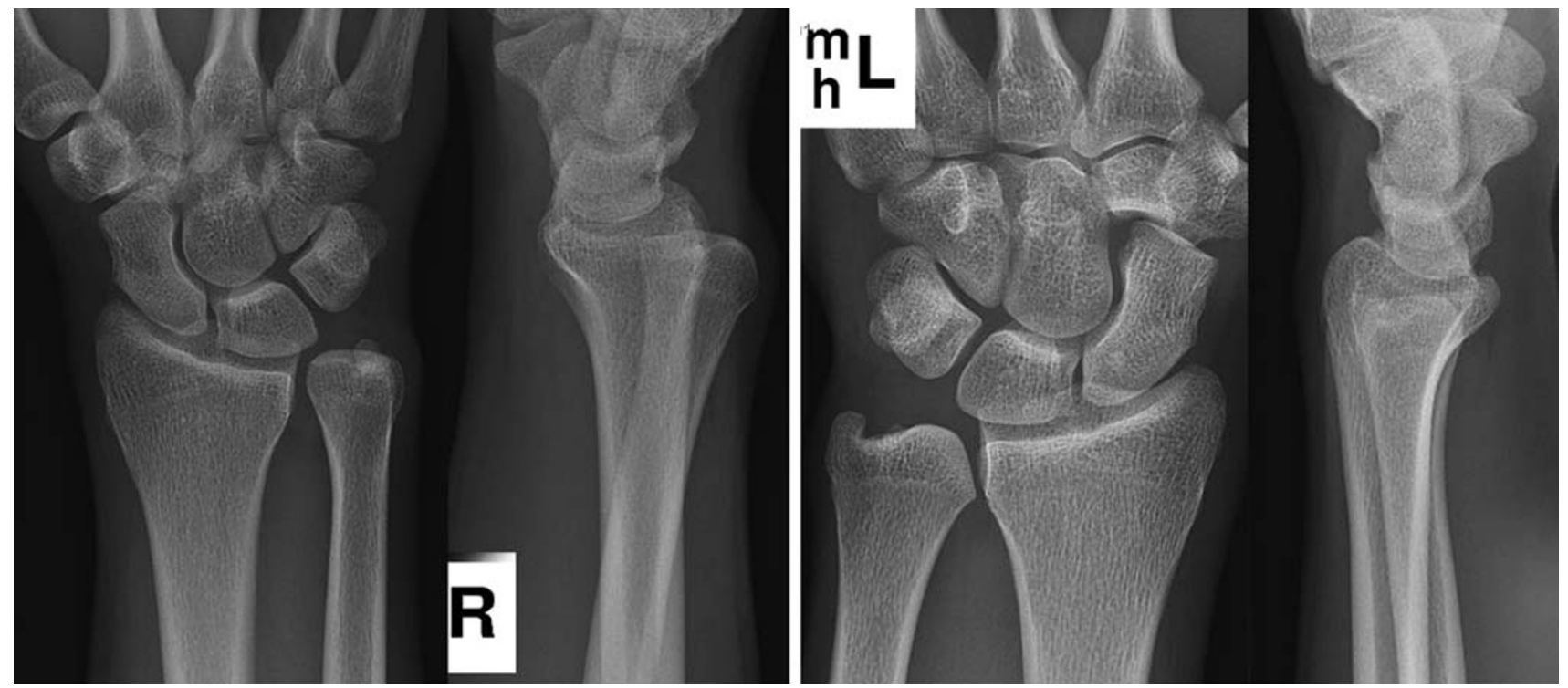

Fig. 1 Left A widening of the distance between ulna and radius can be seen on the anterior-posterior X-ray of the right wrist, the lateral X-ray shows a dorsally displaced distal ulna. Right; the left wrist X-ray made for comparison
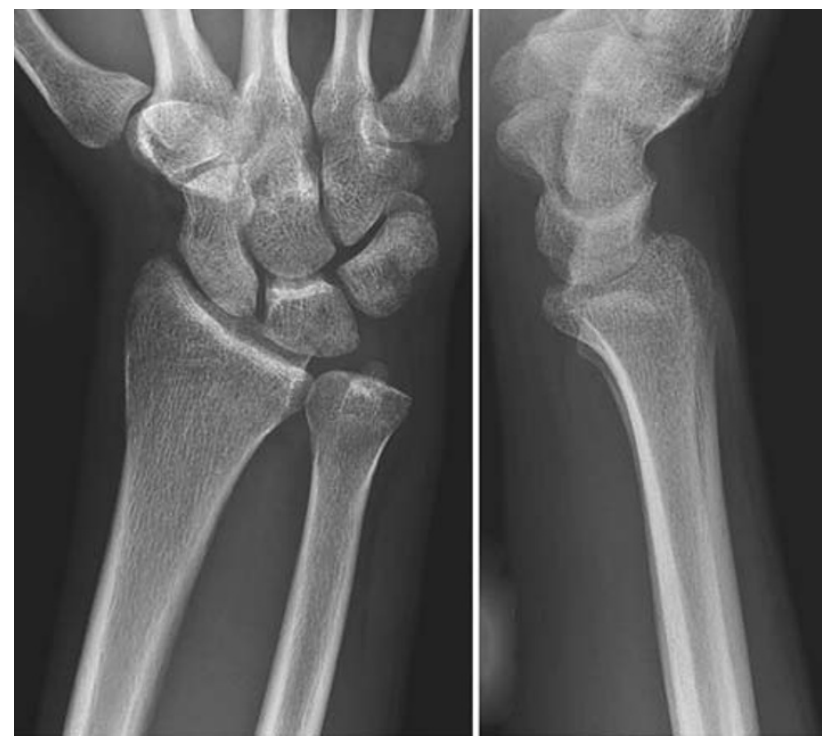

Fig. 2 X-rays of the right wrist at follow up six weeks after dislocation

wrist and protrusion of the ulna dorsally after hitting a punch ball with force. X-ray examination showed recurrence of the isolated DRUJ dislocation. After reduction of the dislocation under conscious sedation, the patient was treated with an above elbow cast in full supination for 6 weeks.

At follow-up, 17 months after the first dislocation and 9 months after the second, he experienced no pain and had no restrictions in work or sports-related activities.

\section{Discussion}

The distal radioulnar joint is the distal articulation in the rotational movement of the forearm allowing only pronation and supination [3]. Since the radius of the curvature of the ulna is $50 \%$ smaller than the radius of the curvature of the radial notch, there is some room for translocation during rotation of the forearm [4]. During supination, the head of the ulna translates palmarly and during pronation dorsally [3]. The radial notch is shallow and does not constrain the ulna during these movements. The triangular fibro cartilage complex (TFCC), the ulnar carpal ligaments, the infratendinous extensor retinaculum, the pronator quadratus muscle and the interosseous membrane provide additional stability [3], with the TFCC being the most important stabilizer.

Fifty percent of all DRUJ dislocations are not recognized at first [5]. The risk of missing a DRUJ dislocation is particularly high if no fracture is present [2]. Unrecognized DRUJ dislocations may lead to secondary instability of the DRU joint [4].

Clinical history and careful physical examination should create a suspicion for DRUJ dislocation. A dorsal DRUJ dislocation presents as a painful wrists after a fall or direct trauma with limited supination or locked pronation. The head of the ulna is prominent dorsally and might show ballottement compared with the unaffected side [3]. Pressure on the distal ulna combined with forceful supination reduce the ulna [3]. Pronation will in most cases dislocate the DRUJ once again. 
A dorsal dislocation of the ulna can occur in three ways. The first is a forced pronation, the second a direct force on the ulna driving it dorsally with fixed radius and carpal bones. The third mechanism of dorsal dislocation is a direct force on the radius driving it palmarly with the ulna held in a fixed position [6].

A standard lateral X-ray can confirm the diagnosis if the $\mathrm{X}$-ray is straight lateral [7]. A mild rotation of the forearm during X-ray examination will alter the position of the ulna in relation to the radius [3, 6]. Nakamura et al. [8] showed that true lateral X-rays are able to detect DRUJ dislocation with almost the same sensitivity as a computed tomography (CT) scan. Some authors suggest a lateral X-ray showing both wrists on a single film [5]. The contralateral wrist should not be suspected of a DRUJ dislocation for comparison. In one case, a bilateral dislocation was described [9]. If uncertainty of the diagnosis persists, a CT scan (preferably) or magnetic resonance imaging (MRI) will show the incongruence of the joint. It might even detect a lesion caused by the dislocation comparable to a Hill-Sachs lesion as is seen in shoulder dislocation [1].

Acute dorsal distal radioulnar joint dislocations are routinely treated with closed reduction and an above elbow cast in supination for 6 weeks. If the dislocation proves to be irreducible even with conscious sedation or general anesthesia, open reduction in combination with repair of the TFCC is recommended [2]. If instability persists, different techniques have been described for operative treatment including the use of a double-breasted slip of the retinaculum [10] and a technique as described by Adams using a tendon graft reconstruction through bone tunnels [4].

In our case, the dorsal dislocation could be reduced under general anesthesia which justifies conservative treatment with cast immobilization. A second traumatic event showed recurrence of the DRUJ dislocation. Once again reduction could be performed, and an above elbow cast was applied. To our knowledge, this is the first report of a recurrent dislocation after successful conservative treatment.

Even if the mechanism is different, this should not affect the result of conservative treatment, as is shown in this case report.

\section{References}

1. Garrigues GE, Aldridge JM III (2007) Acute irreducible distal radioulnar joint dislocation. A case report. J Bone Joint Surg Am 89:1594-1597

2. Dameron TB Jr (1972) Traumatic dislocation of the distal radioulnar joint. Clin Orthop Relat Res 83:55-63

3. Szabo RM (2007) Distal radioulnar joint instability. Instr Course Lect 56:79-89

4. Lawler E, Adams BD (2007) Reconstruction for DRUJ instability. Hand 2:123-126

5. Rainey RK, Pfautsch ML (1985) Traumatic volar dislocation of the distal radioulnar joint. Orthopedics 8:896-900

6. Russo MT, Maffulli N (1991) Dorsal dislocation of the distal end of the ulna in a judo player. Acta Orthop Belg 57:442-446

7. Nicolaidis SC, Hildreth DH, Lichtman DM (2000) Acute injuries of the distal radioulnar joint. Hand Clin 16:449-459

8. Nakamura R, Horii E, Imaeda T, Tsunoda K, Nakao E (1995) Distal radioulnar joint subluxation and dislocation diagnosed by standard roentgenography. Skeletal Radiol 24:91-94

9. Pezeshki C, Weiland AJ (1978) Bilateral dorsal dislocation of the distal radio-ulnar joint. J Trauma 18:673-676

10. Gupta RK, Singh H, Sandhu VP (2008) Stabilisation of the distal radioulnar joint with a double-breasted slip of the extensor retinaculum. J Bone Joint Surg Br 90:200-202 